Document downloaded from:

http://hdl.handle.net/10251/45980

This paper must be cited as:

Escrivá-Escrivá, G.; Ảlvarez Bel, CM.; Roldán Blay, C.; Alcázar-Ortega, M. (2011). New artificial neural network prediction method for electrical consumption forecasting based on building end-uses. Energy and Buildings. 43(11):3112-3119. doi:10.1016/j.enbuild.2011.08.008.

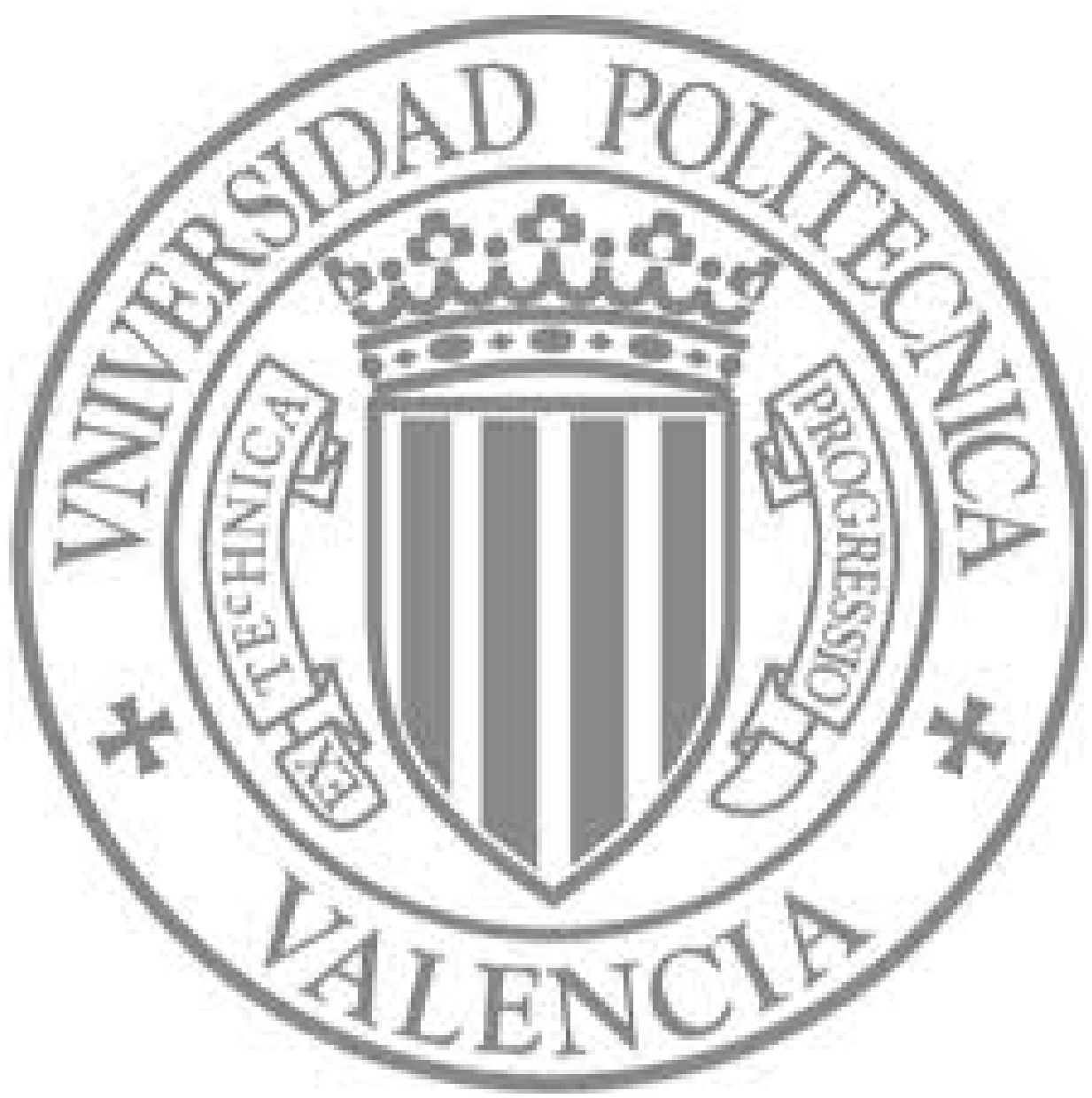

The final publication is available at

http://dx.doi.org/10.1016/j.enbuild.2011.08.008

Copyright Elsevier 


\title{
NEW ARTIFICIAL NEURAL NETWORK PREDICTION METHOD FOR ELECTRICAL CONSUMPTION FORECASTING BASED ON BUILDING END-USES
}

\author{
Guillermo Escrivá-Escrivá $\left({ }^{*}\right)\left({ }^{a}\right)$, Carlos Álvarez-Bel $\left({ }^{a}\right)$, Carlos Roldán-Blay $\left({ }^{a}\right)$, Manuel Alcázar- \\ Ortega $\left({ }^{\mathrm{a}}\right)$
}

${ }^{\left({ }^{a}\right)}$ Institute for Energy Engineering, Universitat Politècnica de València, Camino de Vera, s/n, edificio $8 \mathrm{E}$, escalera $\mathrm{F}, 2^{\mathrm{a}}$ planta. 46022 Valencia, Spain

\begin{abstract}
Due to the current high energy prices it is essential to find ways to take advantage of new energy resources and enable consumers to better understand their load curve. This understanding will help improve customer flexibility and their ability to respond to price or other signals from the electricity market. In this scenario, one of the most important steps is to carry out an accurate calculation of the expected consumption curve, i.e. the baseline. Subsequently, with a proper baseline, customers can participate in demand response programs and verify performed actions. This paper presents an artificial neural network (ANN) method for short-term prediction of total power consumption in buildings with several independent processes. This problem has been widely discussed in recent literature but a new point of view is proposed. The method is based on two fundamental features: total consumption forecast based on independent processes of the considered load or end-uses; and an adequate selection of the training data set in order to simplify the ANN architecture. Validation of the method has been performed with the prediction of the whole consumption expressed as 96 active energy quarterhourly values of the Universitat Politècnica de València, a commercial customer consuming $11,500 \mathrm{~kW}$.
\end{abstract}

Keywords: Building energy consumption; artificial neural networks; building end-uses; forecast method

\footnotetext{
* Corresponding author: Guillermo Escrivá-Escrivá. Institute for Energy Engineering. Universitat Politècnica de València. Camino de Vera, s/n, edificio $8 \mathrm{E}$, escalera $\mathrm{F}, 2^{\mathrm{a}}$ planta, 46022 Valencia (SPAIN). Telf. +34 963879 240; Fax: +34 963877 272, email address: guieses@die.upv.es
} 


\section{Introduction}

Given the current economic situation and the liberalised electricity market, large consumers need to characterise their consumption in order to negotiate lower energy prices, offer flexibility to aggregators, and adapt their consumption pattern [1, 2]. To maximise savings, it is essential to achieve energy efficiency in all processes. Furthermore, any improvement in energy efficiency reduces environmental impact and global warming.

Due to the above reasons it is necessary to take action on energy consumption. To control consumption and achieve further substantial cost savings, it is necessary to study how to achieve greater efficiency. To achieve these savings, consumers must be able to understand their load curve. This may lead to improved customer flexibility and the ability to act in response to price or other electricity market signals [3]. To achieve this and enable users to participate in the electricity markets by offering flexibility, the most important step is to carry out an accurate calculation of the expected consumption curve, i.e. the baseline [4]. Subsequently, with an adequate baseline, customers can participate in demand response programs and verify performed actions.

The forecasting of hourly integrated load carried out for one day to one week ahead is usually referred to as short-term load forecasting [5-7]. There are different methodologies for carrying out short-term forecasting of consumption, for example: autoregressive models; moving average models; linear regression; exponential smoothing; stochastic processes; state space methods; etc. [8-12]. Artificial neural networks (ANNs) and fuzzy systems are two distinct but overlapping attempts to approximate real-world human brain functionality in dealing with multivalued and vague situations. The neural approach requires the specification of a nonlinear dynamic system that is usually feed-forward, the acquisition of a sufficiently representative set of numerical training samples, and the encoding of those training samples in the dynamic system by repeated learning cycles [13]. In this case, the inputs for the ANNS are all factors that may influence in the consumption curve, namely: schedules, temperature, type of day, etc. A proper use of ANN methods achieves better results than other methods - such as simulations in non-linear problems [14]. Moreover, these algorithms have a great versatility, since they can be combined with statistical methods to achieve greater accuracy [15]. 
End-uses (EUs) are the independent energy processes that can be identified and sometimes measured in consumption. Thus, each EU has specific characteristics and a different relationship with inputs.

This approach facilitates various advantages. Firstly, the behaviour of some EUs is monotonous or repetitive, or may be closely linked to one of the input variables, and this enables lower errors. The method studies lower energy consumptions (EUs) and total consumption is calculated as the aggregation of all EUs. Secondly, this technique provides detailed information about each EU, so consumer flexibility can be calculated for each EU with greater accuracy and flexibility strategies can be independently focused on each EU, thus achieving greater efficiency and better use of energy resources.

This paper aims to develop a new method for calculating the consumption forecast based on two fundamental concepts: artificial neural networks (ANNs) applied in its processes (EUs) rather than the total consumption, and a specific and accurate selection of training days. The method is proposed to be used in medium or low aggregation loads.

An application of the proposed method is presented to predict total consumption at the Universitat Politècnica de València (UPV). Two EUs can be highlighted at this consumer processes whose consumption is strongly dependent on temperature; and processes that are temperature-independent.

The paper is organised as follows. Section 2 describes the inputs of the networks and ANN architecture. Section 3 presents the proposed prediction method. Section 4 describes the data set acquisition process performed to acquire the training data. Results and discussion of consumption forecast in the UPV are presented in Sections 5 and 6 respectively. Finally, some conclusions are drawn in Section 7.

\section{Proposed ANN architecture}

This section details the characteristics of the ANN used in the proposed method for predicting consumption in a load. The forecast consists of the calculation of 96 active energy quarter-hourly values for the day of prediction (DOP). The topology presented in this section provides better results for the buildings in which predictions have been made after considering various architectures. 
The pursued objective is to build a neural model capable of adjusting a load curve for a particular facility, and use that model in predicting the power consumption for other days.

The first phase is difficult when developing a neural network model, and is related to the selection of information to feed the ANN during the training phase. Input variables selected as explanatory factors of the load consumption in the considered buildings are discussed below:

- Weather conditions: Possible variables to consider in this category are wind speed, cloudiness, rainfall, temperature, etc. After the analysis of various buildings it has been considered that some of these variables do not significantly affect electrical consumption or appear interrelated with each other. Temperature is the most relevant since it directly affects multiple sources of power consumption such as heat, ventilation, and air-conditioning systems (HVAC), extractors, etc. This relationship between electricity demand and temperature is nonlinear. The proposed ANN architecture considers four variables to characterise these weather conditions: maximum temperature ( $\left.T_{\mathrm{MAX}}\right)$; minimum temperature $\left(\mathrm{T}_{\mathrm{MIN}}\right)$; average temperature ( $\left.T_{A V G}\right)$ of the DOP; and the average temperature of the day before the DOP $\left(T_{A V G-1}\right)$ and which considers system inertia.

- Calendar: Obviously load curve depends on the day and time for which the forecast is made. In the proposed method a different ANN is adjusted for each quarter of hour of the DOP. Accordingly, 96 predictions are calculated to obtain the forecasting daily load curve of a building. The authors consider that consumption depends on the consumption achieved during the days immediately preceding the DOP that meet certain criteria.

- Type of day: Clearly, consumption on a working Monday is not the same as that of a holiday Monday, so it is necessary to consider the type of day. In the proposed method a labour activity parameter (LAP) is used to represent the type of day. This parameter is not considered as an input variable in the ANN, but must be considered in the selection of the training days. Neural networks are trained only with data from days that have the same LAP as the DOP that fit certain criteria.

- Unpredictable factors. There are factors that may affect the consumption, such as a failure of the HVAC system, or another major production failure, strikes, etc. These events should be detected and data must be filtered. 
For model simplicity, only temperature is considered as an input variable of the multi-layer perceptron architecture that has been used as ANN architecture. All other variables are taken into account in other phases of the proposed methodology. Figure 1 shows the chosen ANN architecture.

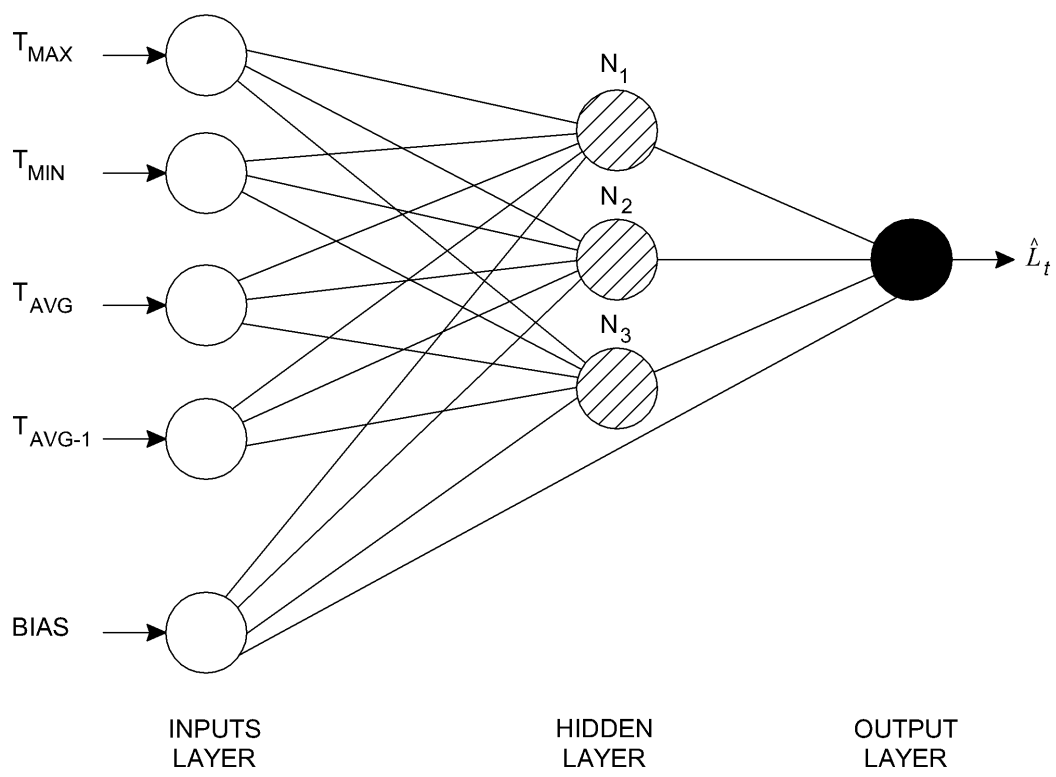

Figure1. ANN architecture used in the proposed method.

The proposed network is composed of three layers: one input; one hidden; and one output layer. ANN architectures with a single hidden layer can represent functions with any form [16], while more layers would assume a greater computational cost.

Four neurons are considered in the input layer: $T_{M A X}, T_{M I N}, T_{A V G}$ and $T_{A V G-1}$.

The output of the neural network has only one neuron, which represents the prediction of the consumption of the plant as quarter-hourly active energy consumption for a given moment $\left(\hat{L}_{t}\right)$. Considering the number of inputs, three neurons were chosen in the hidden layer.

A software tool for the use of the proposed ANN has been implemented in Microsoft Excel 2003, and required macros programmed in Visual Basic for Applications. This application enables the definition of different ANN topologies:

- $\quad$ Number of input variables: from 1 to 50.

- Number of output variables: from 1 to 10.

- $\quad$ Number of neurons in the hidden layer: from 1 to 15.

- Parameter learning: configurable from 0 to 1. 
- Initial value of the weights: configurable from 0 to 1.

- Momentum parameter: from 0 to 1.

- Number of training cycles: up to 500 cycles.

- Training mode: Two modes can be used: batch mode in which all changes are made in the weights of all sets of data at the end of each training cycle; and sequential mode, in which weights change in each set of input data. A learning algorithm called back-propagation (BP) is applied in this work in the sequential mode and the same algorithm is widespread in the literature [17].

Table 1 shows the optimal values for the training parameters, which generally obtain satisfactory results in the studied facilities after trial and error tests. The maximum number of iterations for the calculation of each neural network is 200 , and the iteration with the lowest mean square error (MSE) is selected.

Table 1. Parameter values for the training process.

\begin{tabular}{ll} 
Parameter & Value \\
\hline Cycles & 200 \\
Hidden neurons & 3 \\
Learning parameter & 0.3 \\
Momentum parameter & 0.1
\end{tabular}

\section{ANN prediction method based on EUs}

This paper presents a methodology of calculation for predicting consumption for the next 24-hour period. The prediction consists of 96 values of quarter-hourly active energy consumption. The calculation is made using energy values to avoid the use of instantaneous power values. Average power values may be immediately calculated by multiplying the obtained quarter-hourly values by a factor of four.

Due to the level of aggregation of consumption considered (middle or low consumption loads) the proposed prediction method is based on EUs. Therefore, an ANN may be independently trained for each EU.

Data for each EU may be obtained from: 
- Measurements of each EU. For example, data can be acquired and stored in a data acquisition system (Section 4).

- Simulation of models of different processes.

With the consumption data of the EU and the values of the parameters on which consumption depends (i.e. labour activity parameter of the analysed facilities, external temperatures, etc.) the training of each ANN is performed. Finally, total consumption prediction is obtained using the prediction of the input variables for the DOP and a combination of the predictions of each trained EU (Figure 2).

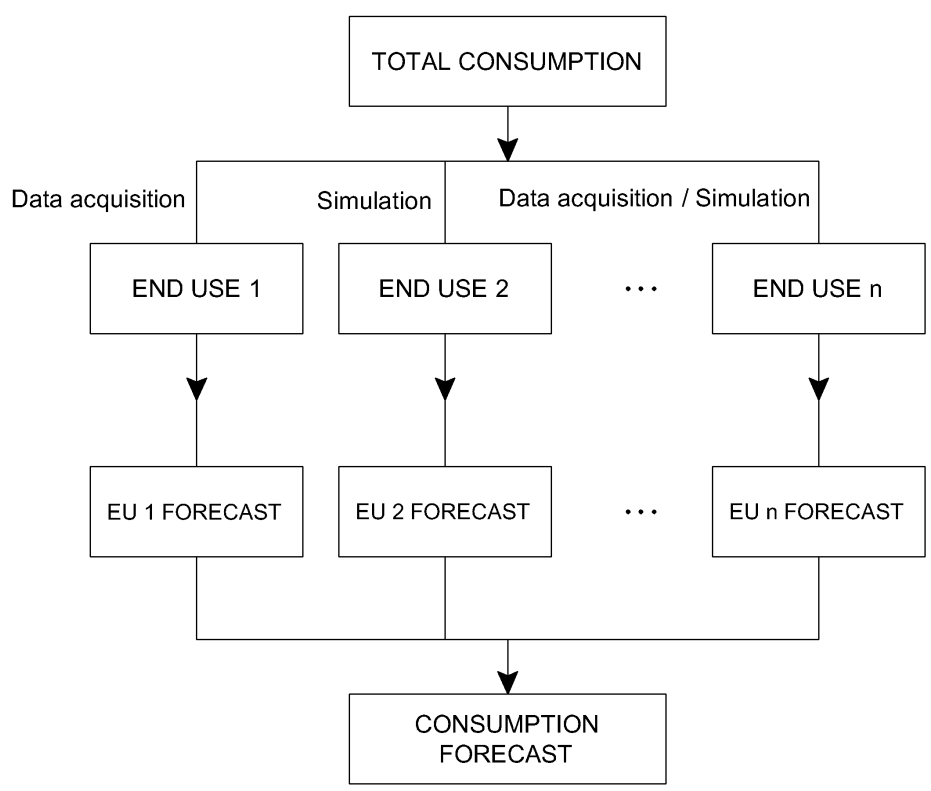

Figure 2. Consumption prediction methodology based on EUs of a building.

Below is a detailed step by step approach for the proposed methodology.

Step 1: Obtaining the value of the input variables for the DOP. Firstly, it is necessary to obtain a prediction of maximum temperature, minimum temperature, and average temperature for the DOP. Furthermore, the average temperature of the day before DOP is used.

Step 2: Selection of training days. For each end-use which breaks down consumption, data is stored to train the ANN. This end-use data, as indicated, can be obtained by measuring the user's premises or through simulations of various processes.

From the available data, which eventually will be very considerable, it is important to select the days that are used to train the ANN. A minimal number of days should be used because of the variability of the data due to the size of the studied loads. 
Each day of consumption is characterised by two parameters: labour activity and CT. The labour activity parameter (LAP) indicates the type and level of activity expected during the day, considering the location, supply point, etc.

The CT parameter is used to characterise days with temperature and is equal to Expression 1.

$$
C T=T_{M A X}+T_{A V G}+T_{M I N}
$$

DOP is assigned with the corresponding LAP value.

In EUs that are strongly dependent on the external temperature, four days that are similar to the DOP are selected to train the ANNs according to Table 2.

Table 2. Training days selection criteria for strongly temperature dependent EUs.

\begin{tabular}{|c|c|c|c|c|c|c|c|c|c|}
\hline Selection criteria & 1 & 2 & 7 & 3 & 4 & 8 & 5 & 6 & 9 \\
\hline Searching days number & 30 & 30 & 30 & 90 & 90 & 90 & 360 & 360 & 360 \\
\hline $\mathrm{CT}+2$ & $x$ & & & $x$ & & & $x$ & & \\
\hline $\mathrm{CT}+1$ & $\mathrm{x}$ & $x$ & & $x$ & $x$ & & $\mathrm{x}$ & $\mathrm{x}$ & \\
\hline CT-1 & $\mathrm{x}$ & $\mathrm{x}$ & $\mathrm{x}$ & $\mathrm{x}$ & $\mathrm{x}$ & $x$ & $\mathrm{x}$ & $\mathrm{x}$ & $\mathrm{x}$ \\
\hline CT-2 & $x$ & $x$ & $\mathrm{x}$ & $x$ & $x$ & $x$ & $x$ & $\mathrm{x}$ & $x$ \\
\hline CT-3 & & $\mathrm{x}$ & $\mathrm{x}$ & & $\mathrm{x}$ & $\mathrm{x}$ & & $\mathrm{x}$ & $\mathrm{x}$ \\
\hline CT-4 & & & $x$ & & & $x$ & & & $x$ \\
\hline $\mathrm{CT}+4$ & & & $x$ & & & $x$ & & & $x$ \\
\hline $\mathrm{CT}+3$ & & $x$ & $x$ & & $x$ & $x$ & & $\mathrm{x}$ & $x$ \\
\hline $\mathrm{CT}+2$ & & $x$ & $x$ & & $x$ & $x$ & & $x$ & $x$ \\
\hline $\mathrm{CT}+1$ & & $x$ & $x$ & & $x$ & $x$ & & $\mathrm{x}$ & $\mathrm{x}$ \\
\hline CT-1 & & $x$ & & & $x$ & & & $\mathrm{x}$ & \\
\hline
\end{tabular}

For example, in the first column for selection criteria (1), the selected days are the four days closest to $\mathrm{CT}$ of the DOP but with higher $\mathrm{CT}$ parameter (namely, $\mathrm{CT}+1, \mathrm{CT}+2$ ) and with lower coefficients (CT-1, CT-2). The days selected must have the same LAP and are taken from the 30 immediately preceding days.

If no data meets this option, then the selection criteria labelled as 2 is chosen. In this case, one day with a higher CT (CT+1), and three days with the next lowest CTs (CT-1, CT-2, CT- 3) are chosen. Again, the selected days must have the same LAP and are taken from the 30 immediately preceding days. 
In other EUs that are not dependent on temperature, the four days nearest the prediction day with the same LAP are chosen to train the ANN.

In the EUs that are slightly temperature-dependent, the training days are chosen from among the preceding 30 days that have the same LAP and CTs closest to the CT of the DOP. If this is not possible, then 90 or 360 days are subsequently considered.

It is important to note that a large amount of data to train the networks should not be used for the type of consumption that arises for the prediction method. The proposed method is for application to small consumers with random consumption - such as a building or a small group of buildings. If many measures are used in the training phase, then random and uncontrolled training data may be introduced. Therefore, a small number of training days with consumption as close as possible to the DOP, and with as much information as possible, are chosen to train the ANNs.

Step 3: Obtaining data on selected days. For the days selected in Step 2 it is necessary to verify that the data is valid because there may be missing data due to problems in the acquisition process, storage, etc. Data is obtained from the database, and if problems are encountered then Step 2 is performed again without considering the days with anomalous data.

For EUs with unavailable measures, data is obtained from the simulation models of the different EUs. Only four simulations are needed for the day temperatures indicated in the preceding step and the same LAP as the DOP. Therefore, for each day selected by CT, required simulations are performed that obtain the active energy consumption for each use with such conditions.

Step 4: Training and validation of the 96 ANN. Thus, for each EU, with selected data according to the criteria above and input variable values for these days, 96 neural networks are trained, one for each quarter-hourly value. Training data for selected days should not be equal, so if necessary a data formatting process is performed by introducing a small random value every day so that data remains unaffected. Validation is performed with the set of days selected following the criteria in Table 2 and not used in the training step.

Step 5: Prediction of the 96 ANN. Some 96 quarter-hourly active forecast energy values for each EU are obtained using the input variable set for the DOP and the 96 trained ANNs. A 
prediction of total consumption is then obtained by the addition of the obtained prediction for each EU.

Step 6: Checking the prediction. It is necessary to establish a method for checking the output of the networks. In this method, consumption in a previous day with similar CT is compared with the total consumption of the obtained forecast values and the deviation must not exceed $10 \%$. If the deviation exceeds $10 \%$, then Step 2 is performed again without considering the days selected previously.

Step 7: Calculation of forecast error. The final step is the calculation of the error of the obtained forecast. Two error indexes are used in this paper to assess the quality of predictions:

- Mean absolute percentage error (MAPE)

This error is commonly used in literature to characterise the goodness of the predictions and is defined by Expression 2:

$$
M A P E=\frac{1}{N} \sum_{t=1}^{N} \frac{\left|\hat{L}_{t}-L_{t}\right|}{L_{t}} \times 100
$$

where:

- $\quad L_{t}$ is the real consumption.

- $\quad \hat{L}_{t}$ is the consumption forecast.

- $\quad N$ is the number of samples.

$M A P E$ is a relative index (without units) that facilitates comparison of the results from different consumers, even if they are obtained for different points of consumption.

- Energy mean error (EME)

This is a new proposed index to characterise the goodness of a calculated forecast and is defined by Expression 3:

$$
E M E=\sum_{t=1}^{N} \frac{\left|\hat{L}_{t}-L_{t}\right|}{\sum_{t=1}^{N} L_{t}} \times 100
$$

$E M E$ index is very similar to the above, but less sensitive in those cases where the real value of consumption is less than 1 . 
Figure 3 shows the steps of the proposed prediction method.

FOR EACH END USE

Day j forecast

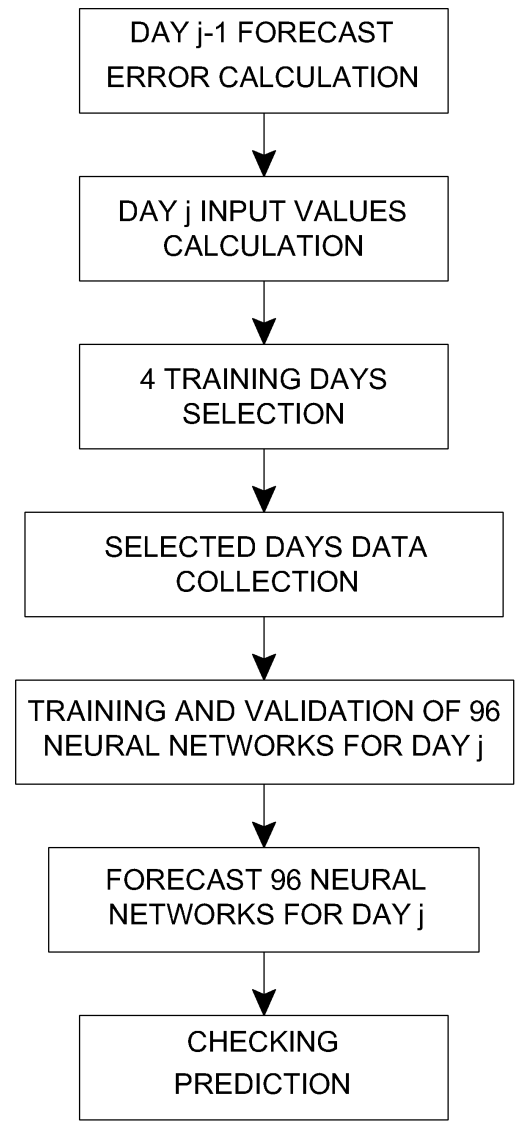

Figure 3. Steps of the proposed prediction method.

The method may be easily integrated in a server application where measurement data is recorded and temperature predictions may be obtained by an Ethernet connection to a weather server.

\section{Training and input data sets}

The manner in which the information required to perform the predictions is collected is shown below. Three sets of data are required. Firstly, consumption values of the days for the training phase and validation of the prediction. Secondly, the external temperature for each day for weather characterisation. And finally, forecast temperature for the DOP.

Obtaining data sets related to consumption to train the ANNs is particularly laborious. In this work, data has been collected using an energy management and control system (EMCS). Using 
the EMCS it is possible to obtain real measures of consumption of the various facilities at the same time, with the advantage of controlling some processes and obtaining precise information of the characteristics of the facilities [18].

A reliable acquisition application is used to avoid data loss. For that purpose, an adequate maintenance of the measurement devices and databases is performed. The existing Ethernet network is used for communication and control purposes, so no specific wiring is necessary. For that reason, installation costs are minimised.

The acquisition application includes the following features:

- The database system is implemented in an SQL server that provides great functionality and adds no major technical constraints to the system.

- Every hour, taking into account their metering intervals, measurement points are checked. If any loss occurs, the system sends a notification via e-mail or through a webpage to the manager of the system.

- Database table size is taken into consideration, so an automatic partitioning is carried out each year.

- Days when the clock goes forward or backward one hour have been considered.

Figure 4 presents the data acquisition architecture that enables consumption measurements to be obtained for several consumers simultaneously.

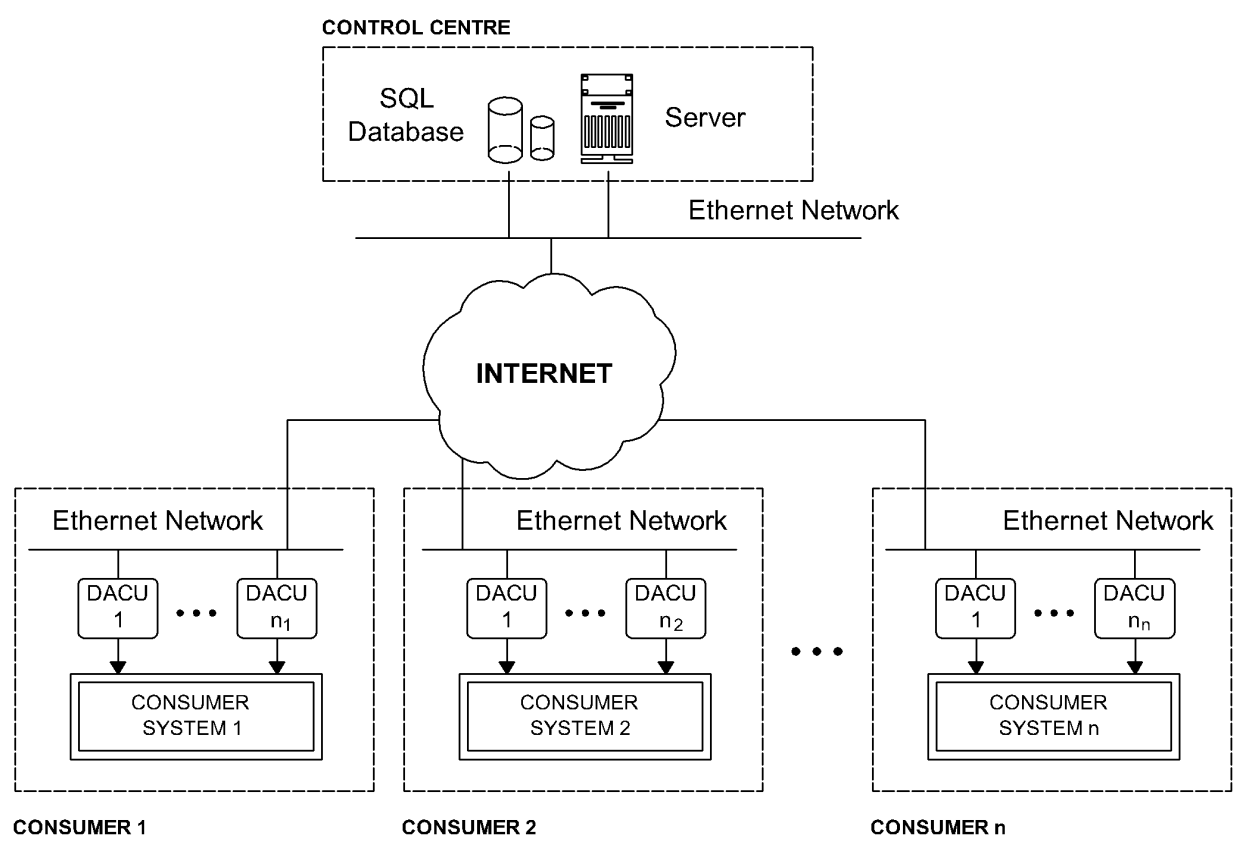

Figure 4. Recommended data acquisition architecture. 
A substantial element in this architecture is the data acquisition central unit (DACU). DACU measures the most relevant electrical magnitudes such as power, currents, and voltages, as well as other measurements related to power quality. The DACU commercial device used is the Merlin Gerin Power Meter 710 [19]. Current transformers $\left(\mathrm{I}_{1} \mathrm{~A} / 5 \mathrm{~A}\right)$ for the particular load are required.

External temperature values have been obtained using data available in historical public databases (for the city of Valencia) $[20,21]$. Temperature forecast for the DOP is obtained through a public weather forecast website [22].

Networks are trained with data for the maximum, minimum, and average temperatures for the DOP and the 96 quarter-hourly active energy values of the selected days. Input data is filtered to identify missing values in electricity consumption and temperature series.

\section{Results}

As an application of the proposed method, consumption forecast for the UPV is presented in this section. The UPV has more 37,000 students, 2,600 lecturers, and 1,700 administrative staff.

The UPV is considered a commercial customer. The contracted capacity is $11,500 \mathrm{~kW}$ and annual energy consumption is about $50 \mathrm{GWh}$, corresponding to about 66 buildings with different uses such as offices, classrooms, laboratories, etc. UPV consumption is dependent on two variables: weather and working patterns.

The labour activity that occurs in the UPV depends on the type of day and the period of the year, so a LAP is accordingly assigned for each day (Table 3):

Table 3. LAP values in UPV depending on type of day.

\begin{tabular}{lcc} 
Type of day & Regular period & Holiday period \\
\hline Monday & 1.00 & 0.78 \\
Tuesday, Wednesday, Thursday & 0.96 & 0.75 \\
Friday & 0.90 & 0.71 \\
Saturday & 0.60 & 0.55 \\
Sunday & 0.50 & 0.48 \\
Day before an official holiday & 0.64 & - \\
Holiday & 0.30 & -
\end{tabular}


Consumption forecast is calculated by using the proposed method with two EUs. End-use 1 denotes all consumption that is strongly temperature-dependent, such as HVAC systems, ventilation systems, and so on. End-use 2 denotes the remaining consumption. LAP is the same for both EUs, since both depend on the type of day (there is a higher consumption of airconditioning and other devices such as lights and PCs when there are more people in the university).

Measured data is only available for total consumption at the UPV, so it is necessary to simulate consumption for each EU (Figure 2). For End-use 2 consumption is calculated extrapolating available data of some buildings with some measured circuits that do not supply air conditioning circuits.

End-use 1 consumption is modelled following the method detailed by the authors in [23]. This consumption has been verified as being very similar to the difference between total consumption (with available measurements) and the modelled consumption for End-use 2. Finally, the End-use 1 is calculated as the difference between total consumption and End-use 2. In this section, the results of two prediction methods are presented to validate the results of the proposed method:

- TC method: Total consumption forecast using the proposed ANN and only using total consumption data .

- EUs method: Total consumption forecast using the proposed method and obtaining the prediction as the aggregation of the different EUs.

Results for Monday, 25 June 2007, are presented in detail. To perform the calculus, values presented in Table 4 are taken as expected values for this date.

Table 4. Data for the calculation of the consumption forecast for 25/06/2007 at the UPV

\begin{tabular}{cccccc} 
LAP & $\mathrm{CT}$ & $\mathrm{T}_{\mathrm{MAX}}$ & $\mathrm{T}_{\mathrm{MIN}}$ & $\mathrm{T}_{\text {AVG }}$ & $\mathrm{T}_{\text {AVG-1 }}$ \\
\hline 1 & $86.60^{\circ} \mathrm{C}$ & $37.61{ }^{\circ} \mathrm{C}$ & $19.95^{\circ} \mathrm{C}$ & $29.05^{\circ} \mathrm{C}$ & $24.12^{\circ} \mathrm{C}$
\end{tabular}

Using the TC method and considering that data for total consumption is temperaturedependent, as indicated in Step 2 of the methodology, the selection of the training days is performed using Table 2. Taking into account the working patterns and the CT, given the 
available data, the selection criteria selected is labelled as 6 (360 days CT+1, CT-3). The training days are: $24 / 7 / 2006\left(\mathrm{CT}=86.88^{\circ} \mathrm{C}\right) ; 31 / 07 / 2006\left(\mathrm{CT}=84.25^{\circ} \mathrm{C}\right) ; 17 / 07 / 2006(\mathrm{CT}=$ $\left.75.83^{\circ} \mathrm{C}\right)$; and $10 / 07 / 2006\left(\mathrm{CT}=73.91^{\circ} \mathrm{C}\right)$.

96 ANNs are trained for each value with the training days and using values from Table 4. Forecasts of the 96 values of active energy quarter-hourly consumption are obtained (Figure 5).

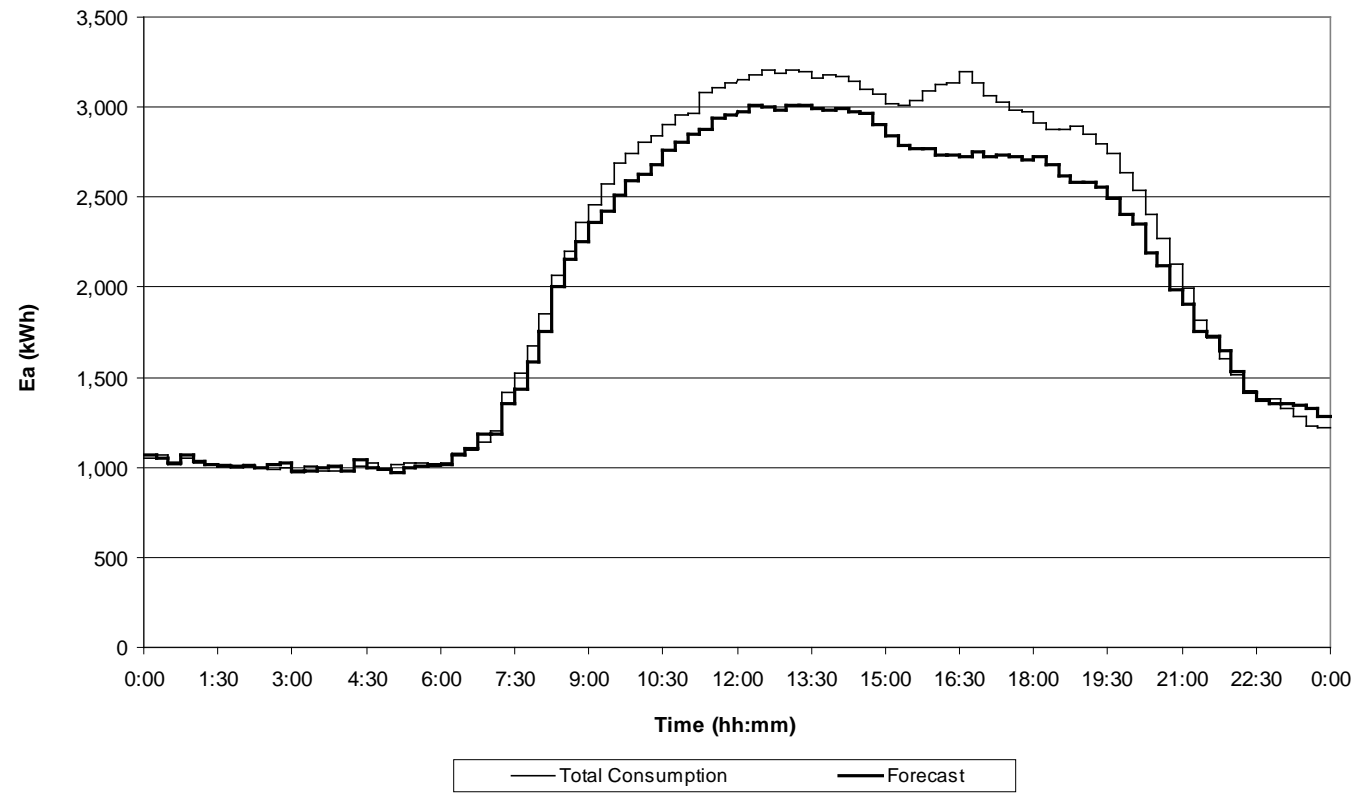

Figure 5. UPV consumption forecast for 25/06/2007 using TC method.

Figure 5 presents the series real total consumption of the UPV (Total consumption) and consumption prediction (Forecast) obtained with the proposed method using total consumption data.

The errors of the prediction are: $M A P E=4.88$ and $E M E=5.95$.

Calculated consumption values are generally lower than the real values because this day is one of the earliest hot days in the year. The available data to calculate the prediction are days from the previous year - although this data does not reflect the annual increase in consumption that occurs because of the growth of the UPV.

This methodology has been implemented in a Web platform that collects the required data and automatically calculates the forecast one day ahead (Figure 6). Consumption data is collected using the architecture presented in Section 4 and the temperature forecast is obtained from a public Web server via file transfer [22]. An application in Visual Basic.Net has been developed to forecast at 3:00 am consumption for the following day using the proposed method. 


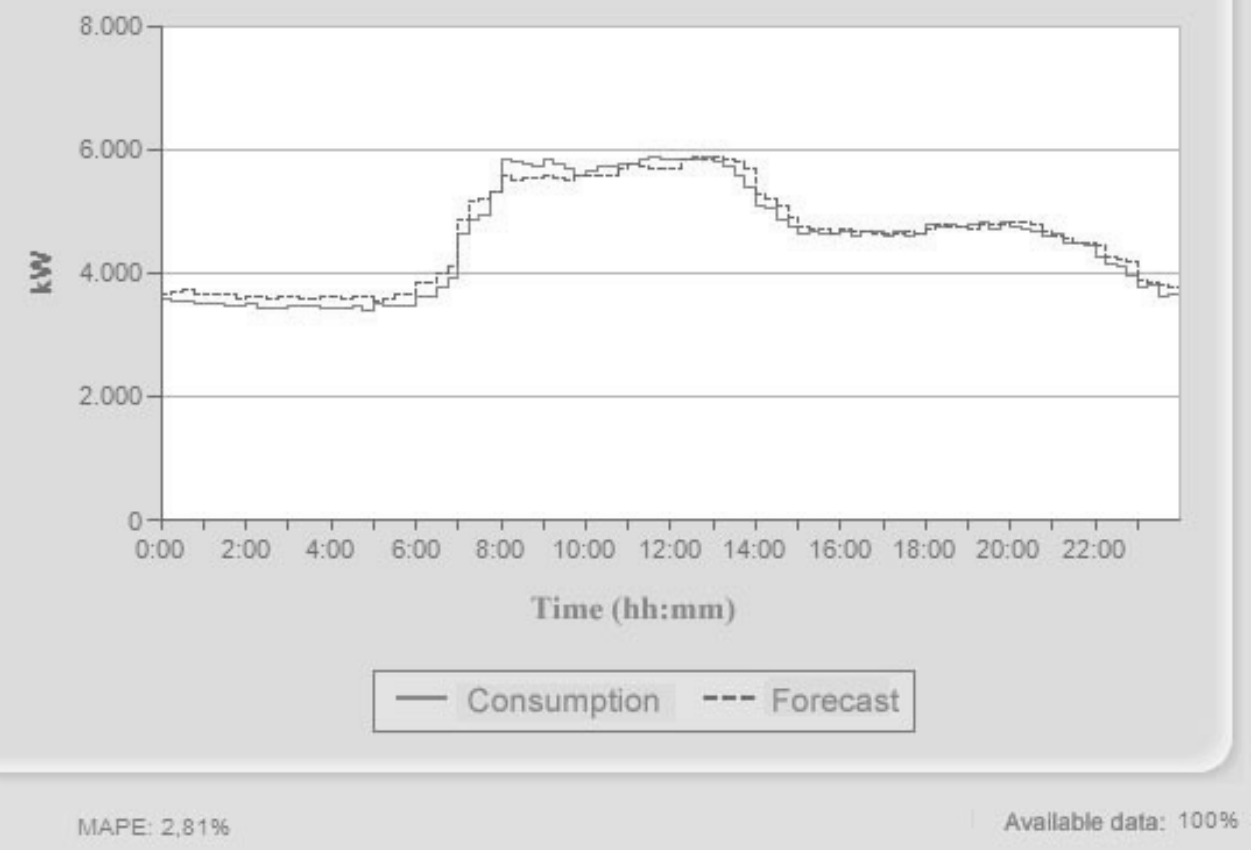

Figure 6. UPV consumption forecast implementation obtained on a Web platform.

Using the EUs method, the consumption forecast is calculated using the proposed method and two EUs. According to Table 2 for End-use 1 the selection criteria is 6, and the training day are the same as above. However, for End-use 2, which is not dependent on temperature, as indicated in Step 2 of the method, the days chosen are those closest to the DOP with the same LAP. The days selected are 18/06/2007, 11/06/2007, 04/06/2007 and 28/05/2007, all of which have LAPs equal to 1.

Prediction is performed for each EU and the forecast is obtained as the aggregation of the prediction of the two EUs. Figure 7 shows the results.

The errors of the prediction are: $M A P E=3.16$ and $E M E=3.36$, and these are significantly lower than those obtained with the previous calculus. 


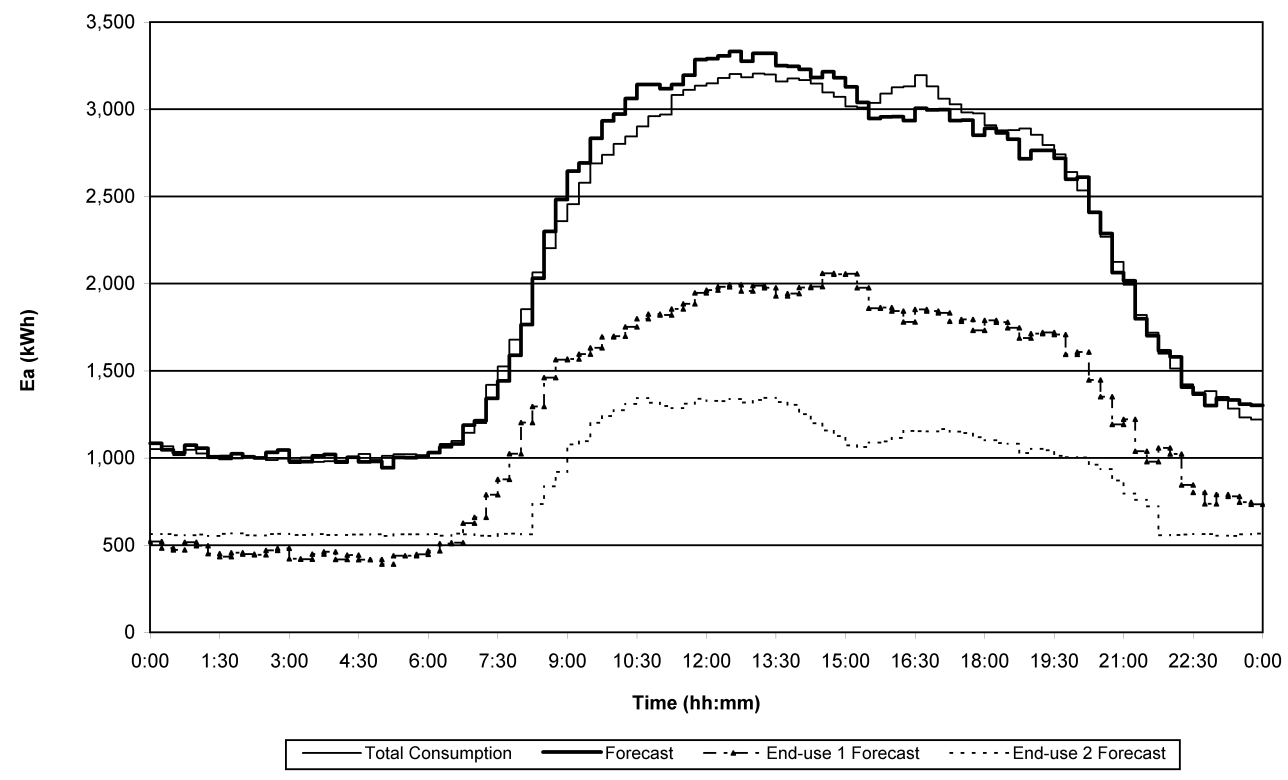

Figure 7. UPV consumption forecast for 25/06/2007 using EUs method.

Figure 8 shows results for the same customer for a full week. In the selected week, Thursday is a holiday, so obtained results for weekdays, Saturdays, Sundays, and public holidays are tested. Satisfactory predictions are obtained for the considered level of aggregation performed in the study.

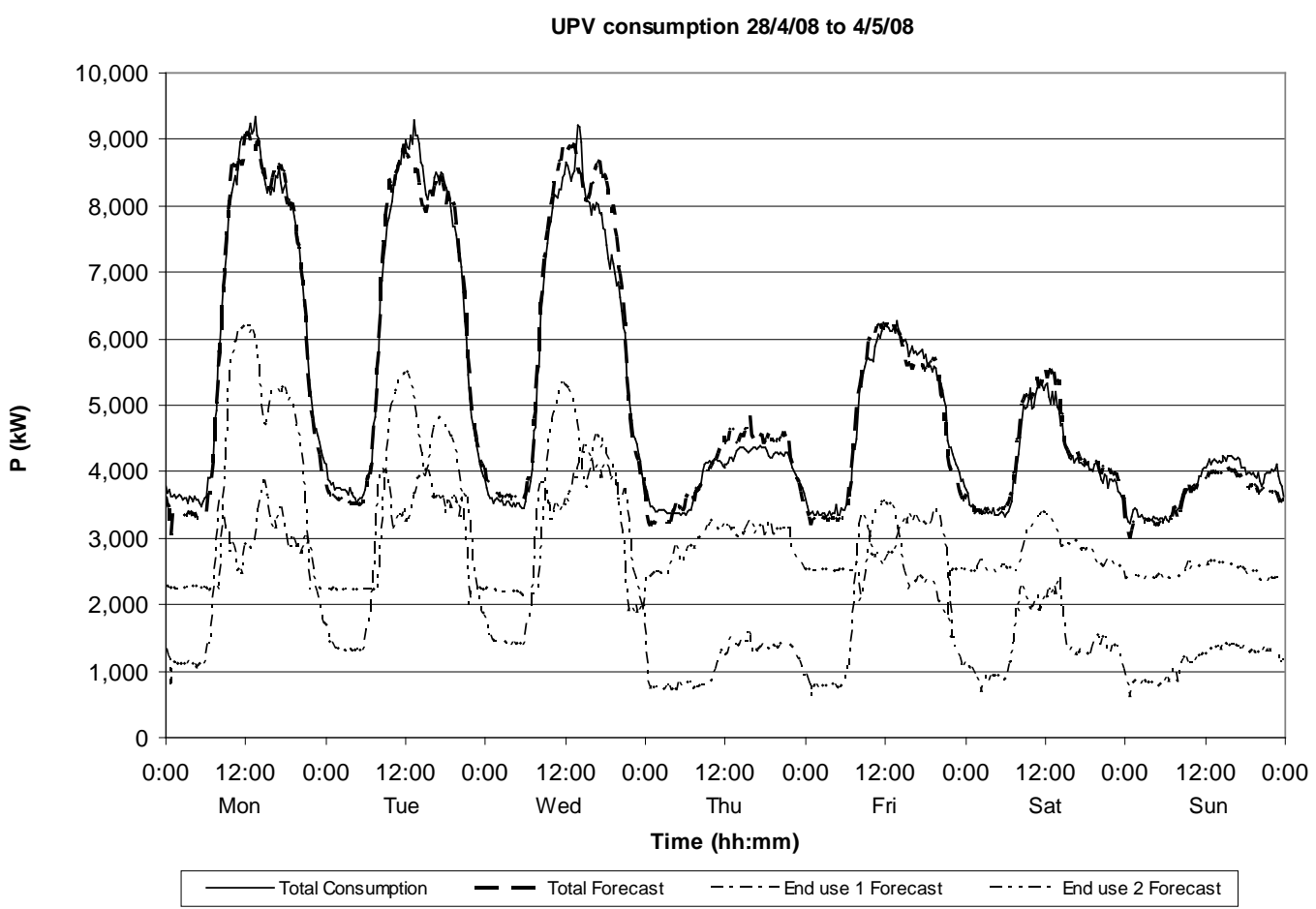

Figure 8. UPV consumption forecast for 28/04/08 to 04/05/08 using EUs method. 
For this period, the forecast errors are $M A P E=3.69$ and $E M E=3.58$. Table 5 shows the errors broken down by day.

Table 5. Forecast errors for UPV consumption forecast for 28/04/08 to 04/05/08 using EUs method.

\begin{tabular}{lccccccc} 
& Mon 28/04 & Tue 29/04 & Wed 30/04 & Thu 01/05 & Fri 02/05 & Sat 3/05 & Sun 04/05 \\
\hline MAPE & 3.39 & 3.00 & 4.41 & 5.42 & 3.13 & 2.59 & 3.90 \\
EME & 2.70 & 2.93 & 4.78 & 5.38 & 3.05 & 2.72 & 4.01
\end{tabular}

450 days were simulated in the UPV and Table 6 shows the results. Predictions obtained with the proposed method based on EUs are satisfactory since the average values of the errors are similar to other methods.

A heuristic method has also been calculated to compare results. The heuristic model calculates the forecast as the Expression 4:

$$
\widehat{L}_{t}=\frac{L_{C T+2+i}+L_{C T+1+i}+L_{C T-1+i}+L_{C T-2+i}}{4}
$$

where $i=0,1,-1, \ldots$ depending on the selection criteria. This method obtains the mean consumption of the four days selected in the TC method, assuming that temperature and behaviour of the systems are similar to the DOP. Despite its simplicity, the heuristic model provides good results, especially on weekdays.

Table 6. Results of multiple simulations at the UPV.

\begin{tabular}{llllllll}
\multirow{2}{*}{ Period } & \multirow{2}{*}{$\begin{array}{l}\text { Days } \\
\text { number }\end{array}$} & \multicolumn{2}{l}{ EUs method } & \multicolumn{2}{c}{ TC method } & \multicolumn{2}{c}{ Heuristic method } \\
\cline { 3 - 7 } & & MAPE & EME & MAPE & EME & MAPE & EME \\
\hline Holiday & 72 & 9.56 & 9.73 & 10.37 & 10.58 & 22.56 & 23.74 \\
Regular & 378 & 5.81 & 5.89 & 6.21 & 6.32 & 7.41 & 7.68 \\
\hline All & 450 & 6.45 & 6.62 & 6.88 & 6.97 & 9.69 & 10.28
\end{tabular}

Table 6 shows that the EU method gives better results than other methods. In the prediction of a holiday with the heuristic method the selected days show significant differences in the temperatures, and therefore in the consumption, and so the forecast obtained is unsatisfactory. 
Table 7. Results of multiple simulations at the UPV in function of the selection criteria in EU's method.

\begin{tabular}{llll} 
Selection criteria & Days number & MAPE & $E M E$ \\
\hline 1 & 111 & 5.10 & 5.30 \\
2 & 61 & 5.16 & 5.27 \\
3 & 92 & 5.81 & 5.89 \\
4 & 46 & 6.42 & 6.68 \\
5 & 112 & 8.65 & 8.91 \\
6 & 11 & 10.82 & 11.18 \\
7 & 7 & 6.04 & 5.99 \\
8 & 8 & 5.08 & 5.37 \\
9 & 2 & 12.17 & 13.42
\end{tabular}

In the EUs method, an analysis of the results shows that the selection criteria with the best results is criteria 1 (Table 7), when considering days very close to the DOP (30 days) and with CTs higher and lower than the DOP. Furthermore, in selection criteria 5 (360 days, CT+2, $\mathrm{CT}+1, \mathrm{CT}-1, \mathrm{CT}-2)$ prediction errors increase - demonstrating that considering more days in the selection process does not produce better results.

A small training data set (with a few days of data) is desirable to improve results in this type of facility. Thus, randomness is avoided and the set of training days is minimised and the selected day conditions (temperature, use of facilities, etc.) are as similar as possible to the DOP.

\section{Discussion}

This section includes a discussion of the basic features of the proposed method:

- The method is based on EUs. This facilitates establishing accurate relationships between inputs and each EU, and the adaptation of the ANNs to the EUs. Furthermore, it is possible to define different inputs for each EU.

- 96 quarter-hourly energy values are obtained for the DOP with little error. The method may be applied to predict any day following a period with data.

- Simple ANNs to minimise the calculation time cost.

- Inputs in the ANN architecture have been reduced to physical variables to avoid introducing too much variability in the model. Other parameters are considered in other steps of the method. 
- Due to the high variability of the considered consumption aggregation level that will be predicted, the ANNs are trained with the minimum number of days, with features as similar as possible to the DOP. Random and uncontrolled training data may be introduced when using many measures in the training phase. Therefore, a small number of training days, but with consumption as close as possible to the DOP and containing as much information as possible, is chosen to train the ANNs.

- The selection of the days is made with two parameters: the labour activity parameter (LAP) to consider work patterns, and weather conditions using the temperature coefficient (CT).

- The average temperature of the day before the DOP is used as input to consider the inertia of the system.

\section{Conclusions}

A new prediction method has been presented using a versatile and adaptive algorithm based on artificial neural networks (ANNs) to predict building electrical consumption. Consumption forecasts are obtained from the prediction of each end-use (EU) of the total consumption. The proposed method provides 96 quarter-hourly energy values of the day of prediction (DOP).

The method is based on EUs in order to establish relationships between inputs and each EU. Other customers could define other inputs.

Due to the high variability of the considered consumption aggregation level, the ANNs are trained with the minimum possible number of days and with features as similar as possible to the DOP. A small number of training days, but with consumption as close as possible to the DOP and containing as much information as possible, is chosen to train the ANNs. The selection of the days is made with two parameters: labour activity parameter (LAP) to consider work patterns, and weather conditions using the temperature coefficient (CT).

As an application of the proposed method, a consumption forecast in the Universitat Politècnica de València (UPV) is presented with very accurate results.

\section{Acknowledgement}

This research work has been possible with the support of the Universitat Politècnica de València (Spain) with grant \#CE 19990032. 


\section{References}

[1] Kathleen Spees, Lester B. Lave. Demand Response and Electricity Market Efficiency. The Electricity Journal, Vol. 20, No. 3, pp. 69-85, April 2007.

[2] International Energy Agency. The power to choose. Demand response in liberalised Markets. IEA publications, 2003.

[3] C. Álvarez-Bel, M. Alcazar-Ortega, G. Escrivá-Escrivá, A. Gabaldón-Marín, Technical and economical tools to assess customer demand response in the commercial sector, Energy Conversion and Management (50) (2009), 2605-2612.

[4] G. Escrivá-Escrivá. New tools to encourage active demand response in competitive electricity markets: implementation and settlement. Dissertation to obtain the degree of doctor, June 2009

[5] Dillon TS, Morsztyn K, Phua K. Short term load forecasting using adaptive pattern recognition and self-organizing techniques. Proceedings fifth world power system computation conference (PSCC-5), Cambridge; 1975, paper 2.4/3, pp. 1-15.

[6] T.S. Dillon, S. Sestito and S. Leung, Short term load forecasting using adaptive neural network, J Electr Power Energy Syst 13 (1991) (4), pp. 186-192.

[7] H.S. Hippert, C.E. Pedreira and R.C. Souza, Neural networks for short-term load forecasting: a review and evaluation, IEEE Trans Power Syst 16 (2001) (1), pp. 44-55.

[8] S.J. Huang and K.R. Shih, Short-term load forecasting via ARMA model identification including non-gaussian process considerations, IEEE Trans Power Syst 18 (2003) (2), pp. 673679.

[9] K. Kim, H.S. Youn and Y.C. Kang, Short-term load forecasting for special days in anomalous load conditions using neural networks and fuzzy inference method, IEEE Trans Power Syst 15 (2000) (2), pp. 559-565.

[10] W. Charytoniuk and M.S. Chen, Very short-term load forecasting using artificial neural networks, IEEE Trans Power Syst 15 (2000) (1), pp. 263-268.

[11] Papalexopoulus AD, Hao S, Peng T. An implementation of a neural network based forecasting model for EMS. IEEE Trans Power Syst 1994, 9 (4), pp. 1956-62.

[12] Chartytoniuk W, Chen MS, Olinda PV. Nonparametric regression based short-term load forecasting. IEEE Trans Power Syst 1998, 13 (3), pp. 725-30. 
[13] J. Acoust. Soc. Am. Neural Networks and Fuzzy Systems. Volume 103, Issue 6, pp. $3131-$ 3131, June 1998.

[14] Alberto Hernandez Neto and Flávio Augusto Sanzovo Fiorellia. Comparison between detailed model simulation and artificial neural network for forecasting building energy consumption. Energy and Buildings. Volume 40, Issue 12, 2008, pp. 2169-2176.

[15] S. Karatasou, M. Santamouris, V. Geros. Modelling and predicting building's energy use with artificial neural networks: Methods and results. Energy and Buildings 38 (2006) 949-958. [16] Denker, J. S., Schwartz, D., Wttner, b., Solla, S., Howard, R., Jackel, L., Hopfield, J. J. Large automatic learning, rule extraction and generalization. Complex Systems, no.1, pp. 877922, 1987.

[17] Gedeon, T. D., Wong, P. M., Harris, D. Balancing the bias and variance: Network topology and pattern set reduction techniques. Proc. int. work on artificial neural networks, IWANN95, pp. 550-8, Torremolinos, Spain, June 1995.

[18] G. Escrivá, M. Alcázar, C. Álvarez. Integral Management System for the energy efficiency improvement in commercial facilities: Application to the Polytechnic University of Valencia. International Conference on Renewable Energies and Power Quality, April 2009. [19] Components specifications. Schneider Electric S. A. Power Logic Manual, 2005. [20] Weather data available on: http://www.wunderground.com/, January 2009. [21] Weather data for different locations, available on: http://www.degreedays.net, February 2009.

[22] Temperature data forecast, available on: http://api.meteored.com, 2010.

[23] G. Escrivá-Escrivá, C. Álvarez-Bel, I. Valencia-Salazar. Method for modelling space conditioning aggregated daily load curves: Application to a university building. Energy and Buildings (42) (2010) 1275-1282. 